\title{
Cognitive and Functional Profiles in Mild-to-Moderate Alzheimer's Disease and Mild Cognitive Impairment Compared to Healthy Elderly
}

\author{
Mark Marsico ${ }^{1}$, Celeste A. de Jager ${ }^{2,3}$, April Grant ${ }^{1}$, Xingshu Zhu ${ }^{4}$, Arwen Markwick², \\ Julie Chandler ${ }^{1}$ \\ ${ }^{1}$ Epidemiology Department, Merck Research Laboratories, North Wales, USA \\ ${ }^{2}$ OPTIMA, Nuffield Department of Medicine, University of Oxford, Oxford, UK \\ ${ }^{3}$ Division of Geriatric Medicine, Department of Medicine, Faculty of Health Sciences, University of Cape Town, \\ Cape Town, South Africa \\ ${ }^{4}$ Statistical Programming, Merck Research Laboratories, North Wales, USA \\ Email: ${ }^{*}$ mark.marsico@merck.com
}

Received 24 October 2014; revised 26 November 2014; accepted 9 December 2014

Academic Editor: Lei Xue, School of Life Science, Tongji University, China

Copyright (C) 2014 by authors and Scientific Research Publishing Inc.

This work is licensed under the Creative Commons Attribution International License (CC BY).

http://creativecommons.org/licenses/by/4.0/

c) (i) Open Access

\section{Abstract}

Background: Amnestic mild cognitive impairment (aMCI) and mild-to-moderate Alzheimer's disease (AD) are clinically distinct but impact cognitive and functional ability similarly. Comprehensive assessment of cognitive and functional deficits may prove useful in informing differential diagnosis in early stages of dementia and in informing endpoint selection in therapeutic AD trials. Objective: The objective of this study was to characterize patterns of cognitive and functional impairment in aMCI and mild-to-moderate AD subjects compared to cognitively intact healthy elderly (HE). Methods: Thirty-one healthy elderly, 20 aMCI and $19 \mathrm{AD}$ participants were administered a cognitive test battery that included the ADAS-Cog and functional assessments. Z-scores were calculated for all endpoints based on the HE reference group. Results: Cognitive deficits were observed in $A D$ and aMCI participants relative to the referent group. On average, aMCI participants performed 1 - 2 standard deviations below HE on cognitive tests, and AD participants performed 2 - 3 standard deviations below HE. Domain-specific functional deficits among AD participants (zscore -0.4 to -6.4 ) were consistently greater than those of aMCI participants (z-score 0 to -1.7 ). Conclusion: This study provides further support for comprehensive assessment and monitoring of

${ }^{*}$ Corresponding author.

How to cite this paper: Marsico, M., de Jager, C.A., Grant, A., Zhu, X.S., Markwick, A. and Chandler, J. (2014) Cognitive and Functional Profiles in Mild-to-Moderate Alzheimer's Disease and Mild Cognitive Impairment Compared to Healthy Elderly. Advances in Alzheimer's Disease, 3, 168-186. http://dx.doi.org/10.4236/aad.2014.34016 
cognitive and functional domain scores in the diagnosis and treatment of aMCI and mild AD. Domain-specific cognitive scores may be more useful than composite scores in characterizing impairment and decline. Measuring domains such as attention, processing speed and executive function may increase the sensitivity of detecting disease progression and therapeutic effects, particularly in mild-moderate AD where memory decline may be too slow to detect drug effects during a typical clinical trial.

\section{Keywords}

Alzheimer's Disease, Amnestic Mild Cognitive Impairment, Dementia, Cognition

\section{Introduction}

Mild Cognitive Impairment (MCI) is characterized by changes in cognition which are less severe and widespread than those of Alzheimer's disease (AD), but represent a decline from cognitive functioning of normal aging and predisposes one to the risk of developing AD [1]. Initially, recognition of MCI was identified by detection of memory performance below age-associated norms [2]. However, recent revisions of this syndrome acknowledge that deficits may also occur in other cognitive domains [3].

Cognitive decline is known to be preceded by neuropathology including beta-amyloid plaque deposition, neurofibrillary tangle formation and atrophy in the brain. Novel research with neuroimaging and CSF markers of pathology has led to revised research criteria for AD diagnosis [4]. The criteria include memory impairment as assessed with sensitive episodic memory tests. This new diagnostic framework has stimulated debate about the definition of AD and related conditions. The Mini-Mental State Examination (MMSE) and Alzheimer's Disease Assessment Scale-Cognitive subscale (ADAS-Cog) have typically been used as outcome measures in AD drug intervention trials [5]; however, these measures may be insensitive for detecting early and subclinical changes [6].

It is widely accepted that the typical cognitive profile in early $\mathrm{AD}$ is marked by episodic memory impairment with semantic memory, working memory, executive functioning, and visuospatial and attentional dysfunction emerging as the disease progresses [7]-[9]. The heterogeneity of cognitive decline suggests endpoints that comprehensively assess cognition may be best suited for detecting cognitive change [10]-[12].

$\mathrm{AD}$ is also marked by deficits in functional ability, characterized by progressive deterioration of activities of daily living (ADL), including early decline in instrumental activities, and later activities relating to basic selfcare [13]. Once loss of ADL occurs and independent living is problematic, diagnosis will include dementia. ADL impairments associated with MCI may occur for instrumental activities that require high-level cognitive skills [14] [15] such as use of technology, understanding cultural expectations [16], managing money [17], medication use [18] and executive functioning [13]. As with cognitive measures, indications of differential profiles of functional impairment have also been found for AD as compared to other dementia groups [19].

Countless cognitive tests have been used worldwide [20] to assess cognitive change in observational studies and clinical trials. In many instances, cognitive tests are included alongside the MMSE and ADAS-Cog in order to evaluate the potential utility of the measures as compared to these "gold standard" trial endpoints. An objective of the Oxford Project to Investigate Memory and Aging (OPTIMA) study was to identify cognitive tests capable of discriminating between cognitively healthy and cognitively impaired groups [21]-[23] as a first step in identifying the tests most likely to identify preclinical $\mathrm{AD}$ and to aid in diagnosis of dementia types. For this study, an adjunct to the original OPTIMA cohort, a selection of cognitive measures was added to the OPTIMA battery with the objective of identifying psychometrically sound cognitive measures for use as cognition endpoints in future $\mathrm{AD}$ clinical trials.

The purpose of this analysis was to determine, in a single cohort of participants, the pattern of cognitive and functional impairment of amnestic MCI-subtype (aMCI) and AD participants, using scores from HE as a reference. Clarification of cognitive and functional profiles is important for accurate differential diagnosis and monitoring decline across dementia syndromes [8] [24]-[27]. Furthermore, comprehensive understanding of domainspecific cognitive and functional profiles in $\mathrm{aMCI}$ and $\mathrm{AD}$ is the first step in identifying particular measures that are sensitive to impairment and the domains that can be utilized as endpoints to better characterize disease and assess impact on the early treatment to mild AD in clinical trials. 


\section{Materials and Methods}

\subsection{Participants}

Participants were recruited from a larger cohort of living subjects enrolled in the Oxford Project to Investigate Memory and Aging (OPTIMA) study within the United Kingdom [28], a longitudinal study of memory and aging established at the University of Oxford. All enrolled subjects undergo a clinical and informant-based interview, a comprehensive cognitive assessment including the Cambridge Cognitive Examination (CAMCOG) [29] [30] and ADAS-Cog, physical examination, MRI brain scan and blood-screening tests as part of the scheduled protocol for diagnostic purposes. Potential participants for the current prospective study were identified from the OPTIMA participant database according to clinical diagnosis at last visit. Those with Mini-Mental State Examination (MMSE) [31] score of $\geq 16(\mathrm{~N}=224)$ had their medical records screened for inclusion/exclusion criteria and rescreened to assess suitability for inclusion in the study. Sixty one subjects were excluded (27\%) based on a review of their medical record. A further 73 (33\%) were excluded after not responding to study invitation letters and telephone calls. The most common reasons for exclusion based on the chart review and telephone screen were: acute illnesses, recent stroke, advanced cancer, other dementia diagnosis than AD, advanced dementia or unwillingness to participate. The remaining 90 subjects $(40 \%)$ were screened by telephone. Current dementia medication and administration of the Telephone Interview for Cognitive Status-Modified (TICS-M) [32] were used to establish a preliminary diagnostic classification.

General inclusion criteria applicable to all subjects for this sub-study, except where noted, were 60 years of age or older, no medical condition affecting cognition (excluding $\mathrm{AD}$ ), a reliable informant willing to act as a study partner (not required for HE), adequate sensory and motor capabilities to perform cognitive testing, fluency in English as determined by the investigator; and the ability to understand study procedures and give verbal consent to participate. Exclusions included: a history of disease that might confound study results, a significant psychiatric history, recent (within 2 years) or current evidence of major untreated depressive or psychiatric disorder, uncontrolled or untreated endocrine disease or other medical condition causing transient or continuous alteration of consciousness or attention, recent (within 2 years) or current evidence of major stroke, multiple lacunar infarcts, transient ischemic events (within 3 months), epilepsy, Parkinson's disease, progressive supranuclear palsy, Huntington's disease, amyotrophic lateral sclerosis, multiple sclerosis or other central neurological disorder or a history of significant head trauma with loss of consciousness.

Final diagnostic classification was made at a consensus meeting of at least two research staff consisting of neuropsychologists and nurses. Cognitive and functional cohort specific diagnostic classification criteria are provided in Table 1.

\subsection{Procedures}

In addition to screening and diagnostic assessments (i.e. Clinical Dementia Rating (CDR) [33], MMSE, Subjective Memory Complaint (SMC) [30] and Geriatric Depression Scale (GDS) [34]), eligible subjects underwent cognitive testing and completed functional and self- and informant-reported assessments at the screening visit. Cognitive and functional data collected at this visit were considered "baseline" data. At subsequent study visits, administrations of cognitive and functional measures were repeated. Information about changes in subject health

Table 1. Cognitive and functional diagnostic classification criteria.

\begin{tabular}{|c|c|c|}
\hline HE & aMCI & AD \\
\hline $\begin{array}{ll} & \mathrm{CDR}=0 \\
\text { - } & \mathrm{MMSE} \geq 28 \\
\text { - } & \text { GDS }<20 \\
\text { - } & \text { TICS-M total score } \geq 29 \\
\text { - } & \text { TICS-m WLR } \geq 11 \\
\text { - } & \text { No significant SMC } \\
\text { - } & \text { Without evidence of depression } \\
\text { - } & \text { Without dementia of ADL impairment }\end{array}$ & $\begin{array}{ll}- & \text { CDR } \leq 0.5 \\
- & \text { MMSE } \geq 23 \\
- & \text { GDS }<20 \\
- & \text { TICS-M total score }<29 \text { and }>14 \\
- & \text { TICS-m WLR }<10 \\
\text { - } & \text { Without dementia and with minimal or } \\
\text { no } & \text { ADL impairment } \\
\text { - } & \text { Met Petersen criteria including the } \\
\text { presence of SMC }\end{array}$ & $\begin{array}{ll}\text { - } & \text { CDR }>0.5 \\
\text { - } & \text { MMSE } \geq 16 \\
\text { - } & \text { GDS }<20 \\
\text { - } & \text { TICS-M total score }<29 \text { and }>14 \\
\text { - } & \text { TICS-m WLR }<10 \\
\text { - } & \text { Possible or probable AD according to } \\
\text { previous or new clinical diagnosis using the } \\
\text { NINCDS-ADRDA criteria }\end{array}$ \\
\hline
\end{tabular}

Abbreviations: CDR: Clinical Dementia Rating; MMSE: Mini-Mental State Examination; GDS: Geriatric Depression Scale; TICS-M: Telephone Interview for Cognitive Status-Modified; WLR: Word List Recall; SMC: Subjective Memory Complaint; ADL: Activities of Daily Living; NINCDSADRDA: National Institute of Neurological and Communicative Disorders and Stroke-Alzheimer's Disease and Related Disorders Association. 
or medical conditions, including changes in medication use and medical events occurring between visits, was collected. The structure of study visits and sequence of study assessments were consistent for all subjects. All study procedures and assessments were administered by either trained research nurses or research neuropsychologists. Only the study's baseline, cross-sectional data are presented in this paper. Longitudinal data collected across subsequent visits ( 6 and 12 months after baseline) are not presented.

Subjects were not administered any marketed or investigational compound in this study. Subjects were however, undergoing usual care by their healthcare providers for their disease which may have included medication indicated for the treatment of $\mathrm{AD}$. Because most subjects began their $\mathrm{AD}$ treatment prior to their inclusion in the study, responsiveness (i.e. the ability of a measure to detect pharmacological change) could not be assessed. All study procedures were in accord with the ethical standards of the Committee on Human Experimentation of the institution in which the experiments were done or in accord with the Helsinki Declaration of 1975. Informed consent was taken on recruitment into the OPTIMA cohort. Ethical approval for this study was granted by the Frenchay Regional Ethics Committee, reference 09/H0107/09.

\subsection{Measures}

Cognitive tests were chosen for this study based on published evidence of the tests' psychometric properties and their usefulness in assessing cognition in AD and aMCI [35]. Tests sensitive to mild impairment were chosen for comparison with the broadly used ADAS-Cog. The overarching aim in selecting cognitive measures was to achieve broad domain coverage with at least two measures assessing each cognitive domain known to be impacted in $\mathrm{AD}$.

The cognitive test battery included the Alzheimer's Disease Assessment Scale-Cognitive subscale (ADASCog) [36]; the Cambridge Automated Neuropsychological Test Battery (CANTAB) [37] Paired Associate Learning (PAL), Reaction Time Index (RTI) and Spatial Working Memory tests; the CAMCOG; and domainspecific tests in verbal and visuospatial episodic memory, semantic memory, attention, executive function and information processing speed (see Table 2). Functional measures included the Alzheimer's Disease Cooperative Study-Activities of Daily Living (ADCS-ADL) [38], scored on basic and instrumental ADL, and the Everyday Memory Questionnaire (EMQ) [39]. Detailed description of all study measures can be found in the online supplement.

\subsection{Statistical Methods}

Standardized z-scores were calculated for all cognitive and functional baseline data based on the mean and standard deviation scores in the HE group. Standardizing the aMCI and mild-to-moderate AD performance to a z-score based on a HE sample allows all test and domain scores to be reported based on the same scale with mean of 0 and a standard deviation of 1 . Domain composite scores were calculated by summing the individual tests' z-score means and dividing by the number of tests comprising that domain. Individual cognitive tests were grouped according to domain as indicated in Table 2. The effects of age and education were assessed using one-way analysis of variance (ANOVA); independent t-tests were conducted to investigate the potential influence of gender. A p-value of $<0.05$ was considered significant for all comparisons. Because cognitive performance was not impacted by any of these covariates (data not shown), unadjusted, raw data were used in all z-score calculations. Because the ADAS-Cog was not administered to HE in this study, standard scores were created using previously published normative data [40] [41].

This study was designed to descriptively assess cognitive performance and functional status in non-demented, aMCI and mild-to-moderate AD. The study was not powered to support formal statistical testing comparing the cognitive and functional outcomes across the three groups. However, statistical tests (ANOVA) were performed and $\mathrm{p}$-values presented to facilitate interpretation of the differences between groups with regard to their cognitive and functional scores. Imputation was not performed for missing data so the number of subjects contributing to group means may vary by endpoint. All statistical analyses were performed using SAS version 9.3.

\section{Results}

\subsection{Demographic Characteristics}

Seventy five OPTIMA subjects prescreened with the TICS-M were invited to come into the study clinic for a 
Table 2. Cognitive and functional test characteristics.

\begin{tabular}{|c|c|c|c|c|c|}
\hline $\begin{array}{c}\text { Cognitive/ } \\
\text { Functional } \\
\text { Battery }\end{array}$ & Test Name & Abbreviation & Cognitive Domain & Outcome Variables & Range \\
\hline \multirow{7}{*}{$\begin{array}{c}\text { CANTAB } \\
\text { Computerized } \\
\text { Battery }^{\dagger}\end{array}$} & $\begin{array}{c}\text { CANTAB Reaction Time 5-Choice Movement } \\
\text { Time }^{*}\end{array}$ & 5cRT Movement & Attention \& Processing & Milliseconds & $0-\infty$ \\
\hline & CANTAB Reaction Time 5-Choice Reaction Time ${ }^{*}$ & 5cRT Reaction & Attention \& Processing & Milliseconds & $0-\infty$ \\
\hline & CANTAB Reaction Time Simple Movement Time ${ }^{*}$ & sRT Movement & Attention \& Processing & Milliseconds & $0-\infty$ \\
\hline & CANTAB Reaction Time Simple Reaction Time* & sRT Reaction & Attention \& Processing & Milliseconds & $0-\infty$ \\
\hline & CANTAB Paired Associates Learning Total Errors ${ }^{*}$ & PALTOTEA & Visuospatial Memory & Total Errors Adjusted & $0-158$ \\
\hline & $\begin{array}{c}\text { CANTAB Spatial Working Memory Between } \\
\text { Errors }\end{array}$ & SWMBE & Working Memory & Errors & $0-326$ \\
\hline & ADAS-Cog Total Score ${ }^{*}$ & ADAS-Cog Total & General Cognition & Total Errors & $0-70$ \\
\hline \multirow{4}{*}{$\begin{array}{l}\text { ADAS-Cog } \\
\text { and } \\
\text { Subtests } \$\end{array}$} & ADAS-Cog Naming Objects and Fingers* & ADAS-cog Objects & Semantic Memory & Total Incorrect & $0-17$ \\
\hline & ADAS-Cog Word Recall ${ }^{*}$ & ADAS-cog Recall & $\begin{array}{l}\text { Verbal Episodic } \\
\text { Memory }\end{array}$ & $\begin{array}{l}\text { Mean Words per Trial Not } \\
\text { Recalled }\end{array}$ & $0-10$ \\
\hline & ADAS-Cog Word Recognition ${ }^{*}$ & $\begin{array}{l}\text { ADAS-Cog } \\
\text { Recognition }\end{array}$ & $\begin{array}{l}\text { Verbal Episodic } \\
\text { Memory }\end{array}$ & Words Not Recognized & $0-24$ \\
\hline & Mazes Seconds ${ }^{*}$ & Mazes & Executive Function & Seconds & $0-240$ \\
\hline \multirow{21}{*}{ OPTIMA $^{\ddagger}$} & Hopkins Verbal Learning Test (Total Recall) & HVLT TR & $\begin{array}{l}\text { Verbal Episodic } \\
\text { Memory }\end{array}$ & Words Recalled & $0-36$ \\
\hline & Hopkins Verbal Learning Test (Delayed Recall) & HVLT DR & $\begin{array}{l}\text { Verbal Episodic } \\
\text { Memory }\end{array}$ & Words Recalled & $0-12$ \\
\hline & The Placing Test & TPT Objects & Visuospatial Memory & Number Correct & $0-10$ \\
\hline & CAMCOG Learning & Learn & Episodic Memory & Number Correct & $0-17$ \\
\hline & CAMCOG Recent Memory & Recent & Episodic Memory & Number Correct & $0-4$ \\
\hline & CAMCOG Remote Memory & Remote & Episodic Memory & Number Correct & $0-6$ \\
\hline & CAMCOG Comprehension & Comprehension & Semantic Memory & Summed Ordinal Scale & $0-9$ \\
\hline & CAMCOG Expression & Expression & Semantic Memory & Summed Ordinal Scale & $0-21$ \\
\hline & Graded Naming Test & GNT & Semantic Memory & Number Correct & $0-30$ \\
\hline & CAMCOG Calculation & Calculation & Working Memory & Number Correct & $0-2$ \\
\hline & Symbol Digit Modalities Test & SDMT & Executive Function & Completed Minus Errors & $0-110$ \\
\hline & Clock Drawing Task 1 & CLOX 1 & Executive Function & Number Correct & $0-15$ \\
\hline & CAMCOG Abstract Thinking & Thinking & Executive Function & Summed Ordinal Scale & $0-8$ \\
\hline & CAMCOG Orientation & Orientation & Orientation & Number Correct & $0-10$ \\
\hline & CAMCOG Perception & Perception & Perception & Number Correct & $0-11$ \\
\hline & CAMCOG Praxis & Praxis & Praxis & Number Correct & $0-12$ \\
\hline & CAMCOG Attention & Attention & Attention \& Processing & $\begin{array}{l}\text { Accuracy \& Summed } \\
\text { Scale }\end{array}$ & $0-7$ \\
\hline & Map Search from the Tests for Everyday Attention & MAP Search & Attention \& Processing & Items Circled & $0-80$ \\
\hline & Letter Comparison Speed & LCS & Processing Speed & Accuracy Score & $0-20$ \\
\hline & Pattern Comparison Speed & PCS & Processing Speed & Accuracy Score & $0-30$ \\
\hline & $\begin{array}{l}\text { Cambridge Cognitive Examination } \\
\text { (CAMCOG total score) }\end{array}$ & CAMCOG Total & General Cognition & Subscales Total Score & $0-107$ \\
\hline \multirow{2}{*}{ ADCS-ADL ${ }^{\sharp}$} & ADCS-ADL Basic Activities of Daily Living & Basic ADL & Functioning & Summed Item Score & $0-18$ \\
\hline & ADCS-ADL Instrumental Activities of Daily Living & Instrumental ADL & Functioning & Summed Item Score & $0-60$ \\
\hline
\end{tabular}




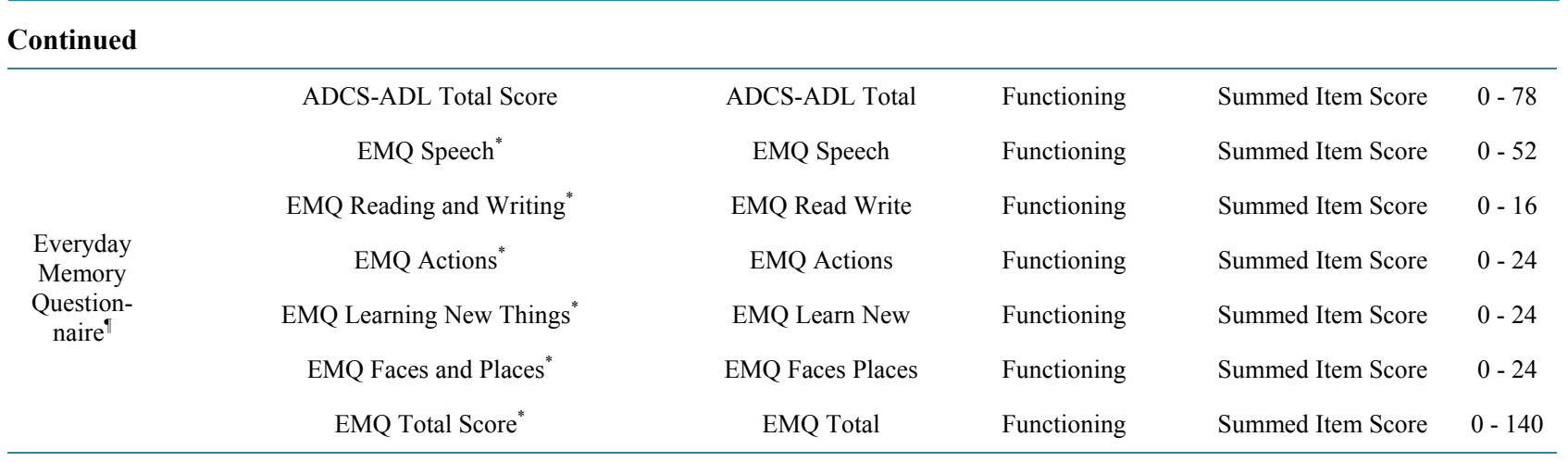

Abbreviations: CANTAB: Cambridge Neuropsychological Test Automated Battery [37]; ADAS-Cog: Alzheimer's Disease Assessment Scale-Cognitive Subscale; OPTIMA: Oxford Project to Investigate Memory and Aging; CAMCOG: Cambridge Cognitive Examination; ADCS-ADL: Alzheimer's Disease Cooperative Study-Activities of Daily Living; EMQ: Everyday Memory Questionnaire. ${ }^{*}$ Higher score equals greater impairment. ${ }^{\dagger}$ Mode of administration is via a Central Processing Unit (CPU). ${ }^{\ddagger}$ Mode of administration is paper and pencil. ${ }^{\S}$ ADAS-Cog normative data was obtained from previous literature. ${ }^{\natural}$ Mode of administration is interview-based.

Table 3. Baseline demographic characteristics and cognitive function scores.

\begin{tabular}{|c|c|c|c|c|}
\hline Baseline Characteristics & $\begin{array}{l}\text { Healthy Elderly } \\
\qquad(\mathrm{n}=31)\end{array}$ & $\begin{array}{l}\text { Amnestic Mild Cognitive } \\
\text { Impairment } \\
(\mathrm{n}=20)\end{array}$ & $\begin{array}{l}\text { Alzheimer's Disease } \\
\qquad(\mathrm{n}=19)\end{array}$ & p Value ${ }^{*}$ \\
\hline Age, mean years $(\mathrm{SD})$ & $80.94(5.47)$ & $80.85(5.38)$ & $77.79(6.90)$ & .15 \\
\hline Gender, males (\%) & $13(41.94)$ & $16(80.00)$ & $11(57.89)$ & .03 \\
\hline Education, mean years (SD) & $14(2.92)$ & $13(2.84)$ & $13(3.00)$ & .63 \\
\hline MMSE, mean score $(\mathrm{SD})^{\dagger}$ & $29.52(0.68)$ & $27.80(1.20)$ & $22.89(2.87)$ & $<.001$ \\
\hline GDS, mean score $(\mathrm{SD})^{\ddagger}$ & $4.10(3.92)$ & $6.40(3.98)$ & $7.50(4.20)$ & .01 \\
\hline Baseline ADCS-ADL Instrumental, mean score (SD) $)^{\S}$ & $53.52(1.82)$ & $52.82(1.91)$ & $42.00(6.42)$ & $<.001$ \\
\hline Baseline EMQ, mean score $(\mathrm{SD})^{\uparrow}$ & $27.10(13.27)$ & $47.10(22.30)$ & $75.95(18.26)$ & $<.001$ \\
\hline
\end{tabular}

Abbreviations: MMSE: Mini-Mental State Examination; GDS: Geriatric Depression Scale; ADCS-ADL: Alzheimer's Disease Cooperative Study-Activities of Daily Living; EMQ: Everyday Memory Questionnaire. *ANOVA was used to calculate p values for continuous variables and chi-square test for categorical variables. ${ }^{\dagger}$ MSE scores range from 0 to 30 , with higher scores indicating less impairment. ${ }^{\ddagger}$ GDS scores range from 0 to 30 , with higher scores indicating more depressive symptoms. ${ }^{\S}$ ADCS-ADL Instrumental scores range from 0 to 60 , with higher scores indicating less impairment. ${ }^{\text {EMQ }}$ scores range from 0 to 140 , with higher scores indicating greater dysfunction.

baseline visit. Only 5 (7\%) participants (all MCI or AD) failed to meet inclusion criteria. The final sample consisted of $31 \mathrm{HE}, 20 \mathrm{aMCI}$ and $19 \mathrm{AD}$.

Results comparing demographic factors by group are presented in Table 3. On average, AD subjects had less education than the HE and were approximately 3 years younger than the HE and aMCI, but the differences were not statistically significant. There were significantly more males recruited into the aMCI and AD groups compared to HE. MMSE scores were statistically different across the groups with highest scores among the HE (29.5 $\pm 0.7)$ and aMCI $(27.8 \pm 1.2)$ compared to $\mathrm{AD}(22.9 \pm 2.9)$. A gradient of increasing functional impairment and depressive symptoms with increasing cognitive impairment was observed between the groups. At baseline, 8 $(42 \%)$ mild-to-moderate $\mathrm{AD}$ subjects had received cognitive-enhancing treatment as part of their usual medical care for an average of $3.9 \pm 3.2$ years.

\subsection{Cognitive Performance}

All means and standard deviations for cognitive test performance in the mild-to-moderate AD, aMCI, and HE groups can be found in Table 4. Figure 1 presents the cross-sectional baseline z-scores for AD and aMCI, grouped by cognitive domain, for each of the cognitive endpoints evaluated. Cut-off lines of 1.5 and 2 standard deviations below the mean are indicated as the usual thresholds of significant deficit from normal control performance for MCI and $\mathrm{AD}$, respectively [2]. All references made to the deficits observed in $\mathrm{AD}$ and aMCI groups are always relative to the HE referent group. Minimum and maximum z-scores within a cognitive do- 
Table 4. Means and standard deviations for cognitive and functional tests.

\begin{tabular}{|c|c|c|c|c|c|c|}
\hline Test Abbreviation & $\mathrm{N}$ & HE Mean \pm SD & $\mathrm{N}$ & $\mathrm{aMCI}$ Mean $\pm \mathrm{SD}$ & $\mathrm{N}$ & $\mathrm{AD}$ Mean $\pm \mathrm{SD}$ \\
\hline $5 \mathrm{cRT}$ Movement ${ }^{*}$ & 31 & $311.32 \pm 100.74$ & 20 & $335.75 \pm 117.27$ & 17 & $425.76 \pm 171.22$ \\
\hline 5cRT Reaction ${ }^{*}$ & 31 & $383.94 \pm 51.94$ & 20 & $466.10 \pm 73.63^{\ddagger}$ & 17 & $491.71 \pm 112.49$ \\
\hline sRT Movement ${ }^{*}$ & 31 & $312.26 \pm 158.18$ & 20 & $311.75 \pm 131.15$ & 17 & $444.00 \pm 213.33$ \\
\hline sRT Reaction ${ }^{*}$ & 31 & $342.65 \pm 65.17$ & 20 & $431.70 \pm 114.87$ & 17 & $466.12 \pm 254.93$ \\
\hline PALTOTEA ${ }^{*}$ & 30 & $37.80 \pm 23.93$ & 19 & $74.53 \pm 33.27^{\ddagger}$ & 18 & $117.94 \pm 18.53$ \\
\hline SWMBE $^{*}$ & 31 & $62.84 \pm 15.76$ & 20 & $25.60 \pm 13.00^{\ddagger}$ & 17 & $30.35 \pm 5.59$ \\
\hline ADAS-Cog Total ${ }^{*}$ & 107 & $5.6 \pm 3.3^{\dagger}$ & 20 & $11.38 \pm 4.78$ & 19 & $24.74 \pm 10.40^{\S}$ \\
\hline ADAS-Cog Objects ${ }^{*}$ & 124 & $0.05 \pm 0.22^{\dagger}$ & 20 & $1.10 \pm 0.97$ & 19 & $2.26 \pm 2.21^{\S}$ \\
\hline ADAS-Cog Recall ${ }^{*}$ & 107 & $2.7 \pm 1.2^{\dagger}$ & 20 & $4.53 \pm 1.30$ & 19 & $6.63 \pm 1.60^{\S}$ \\
\hline ADAS-Cog Recognition ${ }^{*}$ & 107 & $2.0 \pm 2.2^{\dagger}$ & 20 & $4.35 \pm 3.15$ & 19 & $8.16 \pm 3.85^{\S}$ \\
\hline Mazes $^{*}$ & 107 & $30.0 \pm 27.9^{\dagger}$ & 20 & $33.00 \pm 15.50$ & 19 & $40.21 \pm 16.51$ \\
\hline HVLT TR & 31 & $24.35 \pm 4.32$ & 19 & $19.05 \pm 4.56^{\ddagger}$ & 19 & $11.32 \pm 5.20^{\S}$ \\
\hline HVLT DR & 30 & $8.13 \pm 2.29$ & 19 & $5.79 \pm 2.42^{\ddagger}$ & 19 & $0.05 \pm 0.23^{\S}$ \\
\hline TPT Objects & 31 & $9.29 \pm 0.90$ & 19 & $8.58 \pm 1.98$ & 19 & $3.58 \pm 1.87^{\S}$ \\
\hline Learn & 31 & $13.74 \pm 1.79$ & 19 & $11.26 \pm 2.51^{*}$ & 13 & $4.38 \pm 1.45^{\S}$ \\
\hline Recent & 31 & $3.97 \pm 0.18$ & 19 & $3.79 \pm 0.54$ & 13 & $1.92 \pm 1.04^{\S}$ \\
\hline Remote & 31 & $5.45 \pm 0.62$ & 19 & $5.11 \pm 0.88$ & 13 & $3.38 \pm 0.96^{\S}$ \\
\hline Comprehension & 31 & $8.94 \pm 0.25$ & 19 & $8.74 \pm 0.56$ & 13 & $8.31 \pm 0.63^{\S}$ \\
\hline Expression & 31 & $19.52 \pm 1.31$ & 19 & $17.82 \pm 1.07^{\ddagger}$ & 13 & $16.38 \pm 2.87$ \\
\hline GNT & 31 & $26.35 \pm 3.22$ & 19 & $23.26 \pm 4.17$ & 19 & $14.05 \pm 8.39^{\S}$ \\
\hline Calculation & 31 & $1.97 \pm 0.18$ & 19 & $1.79 \pm 0.54$ & 13 & $1.54 \pm 0.66$ \\
\hline SDMT & 12 & $40.33 \pm 9.59$ & 11 & $30.91 \pm 10.63$ & 18 & $24.06 \pm 11.83$ \\
\hline CLOX 1 & 5 & $14.20 \pm 0.45$ & 9 & $12.22 \pm 1.72$ & 12 & $9.67 \pm 3.87$ \\
\hline Thinking & 31 & $7.68 \pm 0.60$ & 19 & $7.16 \pm 1.01$ & 13 & $5.85 \pm 2.12^{\S}$ \\
\hline Orientation & 31 & $9.97 \pm 0.18$ & 19 & $9.53 \pm 0.70$ & 13 & $6.46 \pm 1.85^{\S}$ \\
\hline Perception & 31 & $10.42 \pm 0.85$ & 19 & $10.32 \pm 1.16$ & 13 & $8.54 \pm 2.03^{\S}$ \\
\hline Praxis & 31 & $11.68 \pm 0.48$ & 19 & $10.89 \pm 1.15^{\ddagger}$ & 13 & $10.62 \pm 1.12$ \\
\hline Attention & 31 & $6.68 \pm 0.79$ & 19 & $6.89 \pm 0.32$ & 13 & $6.15 \pm 1.07^{\S}$ \\
\hline MAP Search & 31 & $52.29 \pm 13.86$ & 18 & $43.72 \pm 14.93$ & 19 & $30.05 \pm 16.02^{\S}$ \\
\hline LCS & 10 & $7.70 \pm 2.50$ & 10 & $7.30 \pm 2.06$ & 15 & $5.60 \pm 2.13$ \\
\hline PCS & 10 & $11.60 \pm 1.51$ & 9 & $11.78 \pm 2.59$ & 15 & $8.93 \pm 2.31^{\S}$ \\
\hline CAMCOG Total & 31 & $100.00 \pm 3.49$ & 19 & $93.32 \pm 5.10^{*}$ & 13 & $73.54 \pm 10.13$ \\
\hline Basic ADL & 29 & $22.00 \pm 1.20$ & 17 & $22.00 \pm 1.20$ & 18 & $21.50 \pm 1.20$ \\
\hline Instrumental ADL & 29 & $53.62 \pm 1.82$ & 17 & $52.82 \pm 1.91$ & 18 & $42.00 \pm 6.42^{\S}$ \\
\hline ADCS-ADL Total & 29 & $75.52 \pm 1.82$ & 17 & $74.82 \pm 1.91$ & 18 & $63.50 \pm 7.11^{\S}$ \\
\hline
\end{tabular}


Continued

$\begin{array}{ccccccc}\text { EMQ Speech }^{*} & 31 & 11.35 \pm 6.19 & 20 & 19.30 \pm 9.50^{\ddagger} & 19 & 36.26 \pm 7.03^{\S} \\ \text { EMQ Read Write }^{*} & 31 & 3.10 \pm 2.02 & 20 & 4.95 \pm 3.50 & 19 & 7.05 \pm 3.76 \\ \text { EMQ Actions }^{*} & 31 & 3.48 \pm 3.08 & 20 & 7.20 \pm 4.41^{\ddagger} & 19 & 12.47 \pm 3.96^{\S} \\ \text { EMQ Learn New }^{*} & 31 & 5.94 \pm 2.66 & 20 & 9.05 \pm 2.50^{\ddagger} & 19 & 10.68 \pm 5.98 \\ \text { EMP Faces Places }^{*} & 31 & 3.23 \pm 2.04 & 20 & 6.60 \pm 4.43^{\ddagger} & 19 & 9.47 \pm 4.01^{\S} \\ \text { EMQ Total }^{*} & 31 & 27.10 \pm 13.27 & 20 & 47.10 \pm 22.30^{\ddagger} & 19 & 75.95 \pm 18.26^{\S}\end{array}$

Abbreviations: HE: Healthy Elderly; aMCI: Amnestic Mild Cognitive Impairment; AD: Alzheimer's Disease; 5cRT: Five-Choice Reaction Time; sRT: Simple Reaction Time; PALTOTEA: Paired Associates Learning Total Errors Adjusted; SWMBE: Spatial Working Memory Between Errors; ADAS- Cog: Alzheimer's Disease Assessment Scale-Cognitive Subscale; HVLT TR: Hopkins Verbal Learning Test Total Recall; HVLT DR: Hopkins Verbal Learning Test Delayed Recall; TPT: The Placing Test; GNT: Graded Naming Test; SDMT: Symbol Digit Modalities Test; CLOX1: Clock Drawing Task 1; LCS: Letter Comparison Speed; PCS: Pattern Comparison Speed; CAMCOG: Cambridge Examination for Mental Disorders of the Elderly-Cognitive Examination; ADL: Activities of Daily Living; ADCS-ADL: Alzheimer's Disease Cooperative Study-Activities of Daily Living; EMQ: Everyday Memory Questionnaire. "Higher score equals greater impairment. ${ }^{\dagger}$ ADAS-Cog normative data was obtained from previous literature. ${ }^{\dagger} \mathrm{p}<0.05$ aMCI compared to HE; significance not assessed for ADAS-Cog. ${ }^{\S} \mathrm{p}<0.05$ AD compared to aMCI.

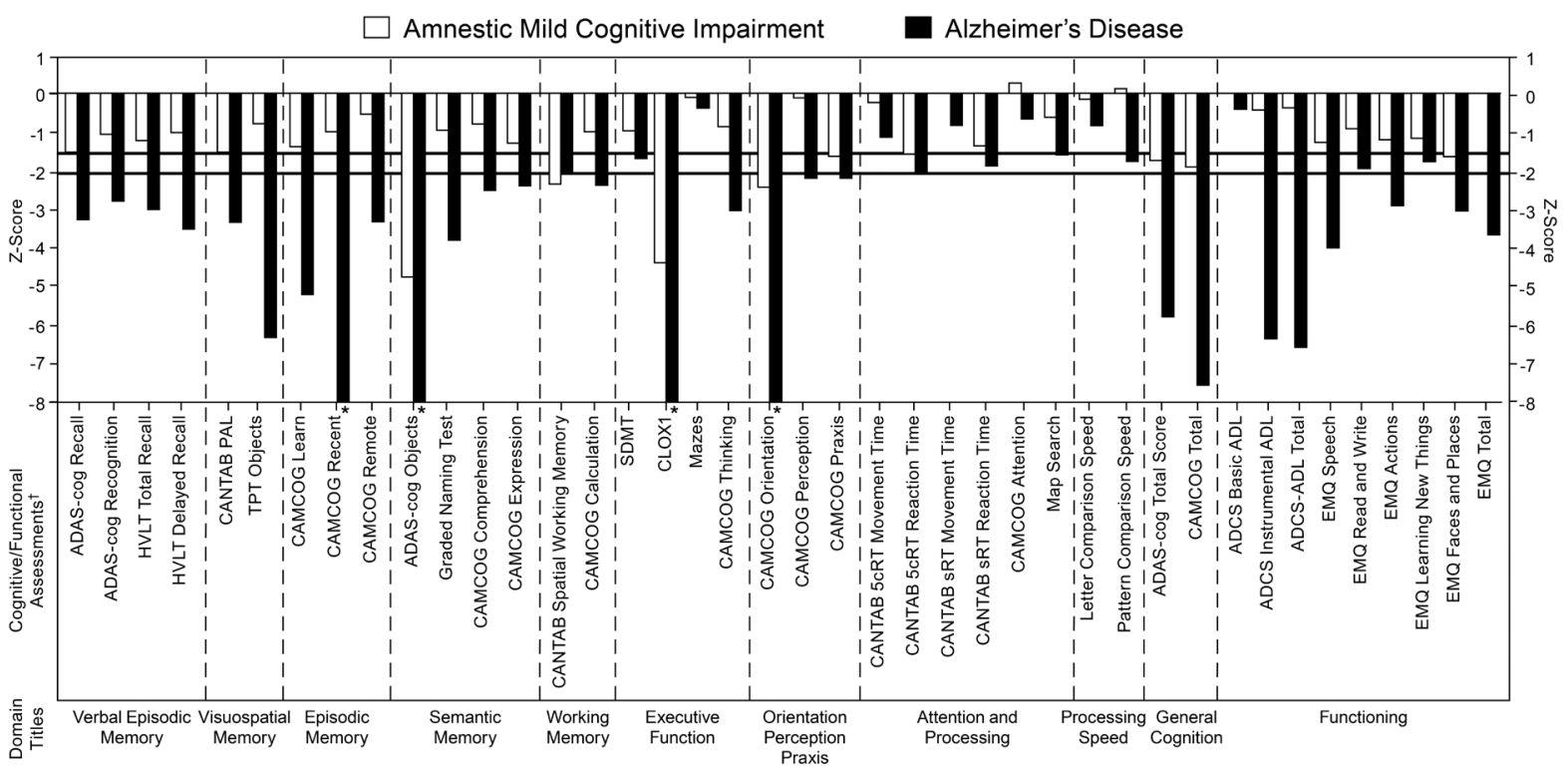

Figure 1. Cognitive and functional z-scores for subjects with mild-to-moderate AD and aMCI normalized to HE. *Actual zscore is greater than -8 . ${ }^{\dagger}$ See Table 2 for information on cognitive and functional assessments, including abbreviations.

main refer to the minimum and maximum scores for the subset of measures classified within each domain. Classifications can be found in Table 2; z-scores are presented in Figure 1.

Deficits were observed among AD subjects relative to aMCI subjects on each cognitive endpoint assessed, with the exception of CANTAB Spatial Working Memory. The greatest cognitive deficits observed in the AD group were within the domains of episodic memory (z-score -3.3 to -11.4 ), executive function (z-score -0.4 to -10.1 ), orientation (z-score -19.5 ), and semantic memory (z-score -2.4 to -10.1 ). Smaller deficits were observed within the domains of processing speed (z-score -0.8 to -1.8$)$ and attention and processing (z-score -0.7 to -2.1). Measureable cognitive deficits were also observed for most endpoints among aMCI with a pattern similar to that seen in the AD group, with the largest deficits in executive function (z-score -0.1 to -4.4 ) and semantic memory (z-score -0.8 to -4.8 ) domains, and the smallest deficits in the processing speed domain (zscores $>-0.2$ ). Endpoints such as the CAMCOG and ADAS-Cog, which utilize a single score to summarize cognitive performance across a variety of domains (categorized in the tables as "general cognition"), detected large differences from $\mathrm{HE}$ in both $\mathrm{AD}$ and $\mathrm{aMCI}$ and were able to clearly differentiate between aMCI (CAMCOG: z-score -1.9; ADAS-Cog: z-score -1.8) and AD (CAMCOG: z-score -7.6; ADAS-Cog: z-score -5.8). Both AD and aMCI had marked deficits in executive function (Clock Drawing Task 1 (CLOX1) [42]; z-scores -10.1 and 
-4.4 ) and semantic memory (ADAS-Cog Object naming; z-scores -10.1 and -4.8). Deficits in verbal episodic memory (ADAS-Cog Recall and ADAS-Cog Recognition) were approximately twice as large in AD (z-scores -3.3 and -2.8$)$ as compared with aMCI (z-scores -1.5 and -1.1$)$, as were those for visuospatial memory (CANTAB PAL; AD z-score -3.4; aMCI z-score -1.5), working memory (CAMCOG Calculation; AD z-score -2.4; aMCI z-score -1.0), semantic memory (CAMCOG Comprehension; AD z-score -2.5; aMCI z- score -0.8 and CAMCOG Expression; AD z-score -2.4; aMCI z-score -1.3), and executive function (Symbol Digit Modalities Test (SDMT) [43]; AD z-score -1.7; aMCI z-score -1.0). Both AD and aMCI had deficits compared to HE in CANTAB Reaction Time tasks (AD z-score -1.9 to -2.1 ; aMCI z-score -1.4 to -1.6), MAP Search (AD z-score -1.6; aMCI z-score -0.6) [44], CANTAB Spatial Working Memory (AD z-score -2.1; aMCI z-score -2.4) and CAMCOG Praxis (AD z-score -2.2; aMCI z-score -1.6).

Figure 2 displays mean composite z-scores by cognitive domain for domains that were assessed by at least 2 tests.

\subsection{Functional Status}

All means and standard deviations for functional measures in the $\mathrm{AD}$, aMCI, and $\mathrm{HE}$ groups can be found in Table 4. Figure 1 also presents the cross-sectional baseline group z-scores for the AD and aMCI groups for each of the composite and individual functional endpoints evaluated.

The ADCS-ADL and EMQ total scores summarize functional status across several domains using a composite score. Measurable deficits relative to HE were observed in both AD (ADCS-ADL: z-score -6.6; EMQ: z-score -3.7) and aMCI (ADCS-ADL: z-score -0.4; EMQ: z-score -1.5). Domain-specific functional deficits were also detected; the deficits were greater among $\mathrm{AD}$ (z-score -0.4 to -6.4 ) compared to aMCI (z-score 0.0 to -1.7). There were no deficits observed in ADCS-ADL Basic ADLs for aMCI compared to HE, but impairment was seen in the ADCS-ADL's instrumental items. The mean instrumental ADL deficit in AD (z-score -6.4) was 16-fold greater than in aMCI (z-score -0.4). Both AD and aMCI had large deficits compared to HE in EMQ sub-domains (AD z-score -1.8 to -4.0 ; aMCI z-score -0.9 to -1.7 ). Functional deficits on the EMQ Total Score were more than twice as large in $\mathrm{AD}$ (mean z-score -3.7) compared to aMCI (mean z-score -1.5).

\section{Discussion}

Although both the cognitive and functional deficits in mild-to-moderate AD have been well-documented in the literature, the deficits have rarely been so well characterized and in a single cohort of participants that spans the spectrum of cognitively intact $\mathrm{HE}, \mathrm{aMCI}$ and mild $\mathrm{AD}$. The cognitive and functional profiles presented in this study provide insight into the comprehensive impairment among aMCI and mild AD.

Detailed characterization of domain-specific cognitive and functional profiles in aMCI and mild-to-moderate $\mathrm{AD}$ populations plays an important role in identifying domains whose exploration may improve the sensitivity of detecting therapeutic effects in clinical trials; albeit, identification of domain-specific measures for use in

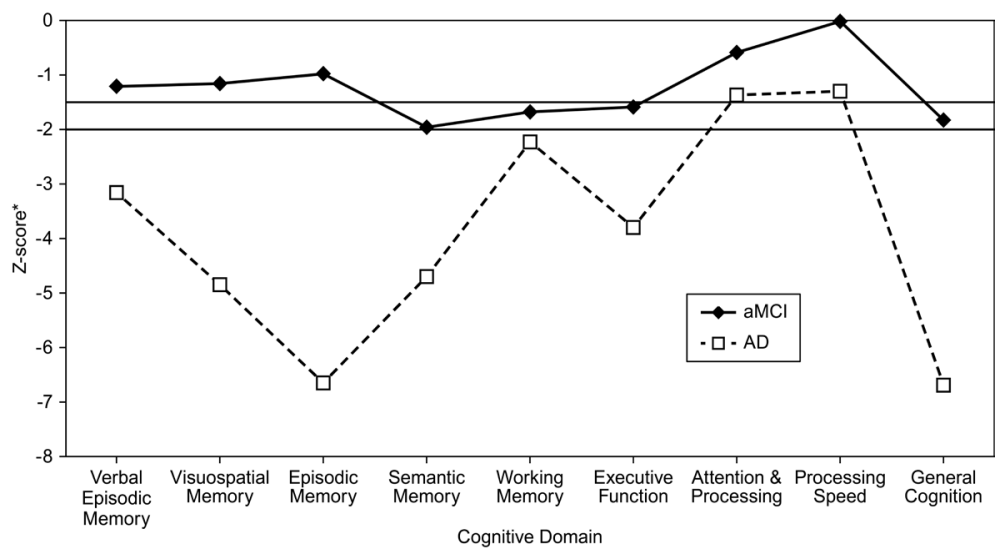

Figure 2. Mean composite z-scores by domain, aMCI and mild-to-moderate AD subjects. ${ }^{*} \mathrm{z}$-scores represent a composite of all the study's tests that assessed the domain listed. 
clinical trials requires further evaluation of the psychometric properties of the tests (i.e. reliability, construct and known-groups' validity, sensitivity to detect longitudinal change). A better understanding of expected cognitive and functional deficits across the spectrum of $\mathrm{AD}$ can also aid in improving the accuracy of diagnosing early and late stages of disease and differentiating between dementia subtypes [8] [21] [24]-[27].

Measurable cognitive and functional deficits were observed in aMCI compared to HE. Despite some overlap in the distribution of individual subject scores, on average, aMCI subjects performed $1-2$ standard deviations below HE on cognitive tests and within 1 standard deviation on functional tests. The aMCI deficits were markedly less than those observed in AD. Although only a proportion of the aMCI subjects are expected to progress to $\mathrm{AD}$, the consistency of their deficits suggests widespread impairment in cognition and function is present years before a clinical diagnosis of AD. Our data corroborates previous research suggesting that the most precipitous domain-specific decline on the continuum from MCI to AD conversion usually takes place in episodic memory [7]-[9].

The cognitive deficits observed in the mild-to-moderate $\mathrm{AD}$ group are consistent with previous research demonstrating the greatest differences from HE present in the domains of visuospatial memory (z-score -3.4 to -6.3 ), episodic memory (z-score -3.3 to -11.4 ), semantic memory (z-score -2.4 to -10.0 ) and executive function (z-score -0.4 to -10.1) [7]-[9] [21] [41]. The consistency of the deficits in attention and information processing in aMCI and AD suggest that deficits in higher-level cognitive processes (e.g. memory, executive function) may be associated with a fundamental attention deficit that manifests very early in the disease. The data presented here support prior experience indicating that domains other than episodic memory are compromised in aMCI or preclinical AD [45]-[47].

Based on the data presented, ADAS-Cog Immediate Recall and Recognition items, the Hopkins Verbal Learning Test revised (HVLT) Immediate and Delayed Recall [48], CAMCOG Calculation, Comprehension and Expression sub-scores, CANTAB PAL Total Adjusted Errors and the SDMT show the most promise as trial endpoints as they exhibit the ability to differentiate between HE, aMCI and AD and are appropriately scaled for these populations. General cognition scores such as the CAMCOG and ADAS-Cog total scores would appear to be less useful clinical trial endpoints since changes in total scores are more difficult to map to the underlying domain-specific impairment. Furthermore, most of the ADAS-Cog items are inappropriately scaled for use in mild $\mathrm{AD}$ and therefore lack the sensitivity to measure widespread domain specific impairment. As a result, in mild AD, the ADAS-Cog total score is an endpoint primarily impacted by only a couple of verbal episodic memory items (namely Word Recall and Recognition). Consequences of excluding impacted domains in a global cognition endpoint include underestimating overall impairment and the difference between mild and moderate $\mathrm{AD}$ and missing clinical change in mild patients when it occurs [49].

Functional decline was previously thought to be a feature that distinguished those with AD from those with more mild impairment, such that early criteria for MCI indicated that one should demonstrate "normal activities of daily living" [50]. However, as with cognitive criteria, recent revisions have noted that there may be subtle but detectable changes in functional ability in MCI as well [51].

Our data indicate a subtle but consistent impairment of functional activities in aMCI, particularly in the EMQ functional activities that correlate highly with memory performance. Activities that require use of high-level cognitive skill, including reasoning, planning, organization, and initiation abilities are more likely to be impacted in aMCI [13], perhaps because these kinds of activity are less routine and well-learned [16]. In the AD group, the instrumental ADCS-ADL and EMQ Speech domain impairment indicated is consistent with cognitive dysfunction in executive, orientation and episodic memory domains. Executive dysfunction has previously been associated with Instrumental ADL impairments [52].

A number of limitations should be considered when interpreting these results. Impairment observed in this study may not be representative of a larger and more culturally diverse sample of subjects with aMCI and mildto-moderate AD. Recruitment of a convenient community sample of well-educated Caucasians and the study's small sample size may limit the generalizability of our findings to demographically and clinically similar populations. A major strength of the study is that it was conducted at a single study site with highly experienced neuropsychologists and nurses trained in cognitive assessments. It should however be acknowledged that the use of a single site with very qualified test administrators, which ensures high data quality and minimal loss to follow up, likely resulted in less performance-related variability than what might be observed in a multi-site, multicountry clinical trial setting.

Standard scores normalized to a healthy elderly population were used to allow for simple comparison of cog- 
nitive and functional performance across domains. It should, however, be noted that some z-scores were generated using non-normally distributed normative data. On a number of the tests the confluence of a compressed range of performance and ceiling effects (i.e. a meaningful number of subjects scoring at the upper limit of a scale's range) among healthy elderly resulted in z-scores among mild-to-moderate AD that may not accurately reflect the true percentage of scores that fall below a given z-score value.

A cross-sectional analysis limits the ability to assess key psychometric properties of the tests, such as test-retest reliability, the presence and persistence of learning effects and the sensitivity to change due to cognitive worsening. Data on these properties is necessary to determine the appropriateness of the endpoints for clinical trials. Future analyses of these data will include comprehensive psychometric analysis of each of the study's tests including their longitudinal performance in each of the study's cohorts.

\section{Conclusion}

The results of this study provide further support for comprehensive assessment and monitoring of cognitive and functional domain scores in the diagnosis and treatment of aMCI and mild-to-moderate AD. Establishing cognitive and functional profiles and assessing their change over time may inform differential diagnosis, particularly as cognitive decline is expected to be reflected in, or correlated with functional decline [53]. Domain-specific cognitive scores may be more useful than composite total scores in identifying impairment and decline in cognitive function related to regional brain function. Measuring domains such as attention, processing speed and executive function may increase the sensitivity of detecting disease progression and therapeutic effects, particularly in mild-to-moderate $\mathrm{AD}$ patients whose memory decline may be too slow to detect drug effects during a typical clinical trial. Further exploration of patterns of impairment may also have potential to be predictive of those at greatest risk of disease progression or who may benefit most from treatment.

\section{References}

[1] Levey, A., Lah, J., Goldstein, F., Steenland, K. and Bliwise, D. (2006) Mild Cognitive Impairment: An Opportunity to Identify Patients at High Risk for Progression to Alzheimer's Disease. Clinical Therapeutics, 28, 991-1001. http://dx.doi.org/10.1016/j.clinthera.2006.07.006

[2] Petersen, R.C., Doody, R., Kurz, A., Mohs, R.C., Morris, J.C., Rabins, P.V., et al. (2001) Current Concepts in Mild Cognitive Impairment. JAMA Neurology, 58, 1985-1992. http://dx.doi.org/10.1001/archneur.58.12.1985

[3] Artero, S., Petersen, R., Touchon, J. and Ritchie, K. (2006) Revised Criteria for Mild Cognitive Impairment: Validation within a Longitudinal Population Study. Dementia and Geriatric Cognitive Disorders, 22, 465-470. http://dx.doi.org/10.1159/000096287

[4] Dubois, B., Feldman, H.H., Jacova, C., Dekosky, S.T., Barberger-Gateau, P., Cummings, J., et al. (2007) Research Criteria for the Diagnosis of Alzheimer's Disease: Revising the NINCDS-ADRDA Criteria. Lancet Neurology, 6, 734746.

[5] Birks, J. (2006) Cholinesterase Inhibitors for Alzheimer's Disease. The Cochrane Database of Systematic Reviews, Article ID: CD005593.

[6] Markwick, A., Zamboni, G. and de Jager, C.A. (2012) Profiles of Cognitive Subtest Impairment in the Montreal Cognitive Assessment (MoCA) in a Research Cohort with Normal Mini-Mental State Examination (MMSE) Scores. Journal of Clinical and Experimental Neuropsychology, 34, 750-757. http://dx.doi.org/10.1080/13803395.2012.672966

[7] Bondi, M.W., Jak, A.J., Delano-Wood, L., Jacobson, M.W., Delis, D.C. and Salmon, D.P. (2008) Neuropsychological Contributions to the Early Identification of Alzheimer's Disease. Neuropsychology Review, 18, 73-90. http://dx.doi.org/10.1007/s11065-008-9054-1

[8] Karantzoulis, S. and Galvin, J.E. (2011) Distinguishing Alzheimer's Disease from Other Major Forms of Dementia. Expert Review of Neurotherapeutics, 11, 1579-1591. http://dx.doi.org/10.1586/ern.11.155

[9] Nordlund, A., Rolstad, S., Hellstrom, P., Sjogren, M., Hansen, S. and Wallin, A. (2005) The Goteborg MCI Study: Mild Cognitive Impairment Is a Heterogeneous Condition. Journal of Neurology, Neurosurgery and Psychiatry, 76, 1485-1490. http://dx.doi.org/10.1136/jnnp.2004.050385

[10] Caine, D. and Hodges, J.R. (2001) Heterogeneity of Semantic and Visuospatial Deficits in Early Alzheimer's Disease. Neuropsychology, 15, 155-164. http://dx.doi.org/10.1037/0894-4105.15.2.155

[11] Carter, S.F., Caine, D., Burns, A., Herholz, K. and Ralph, M.A.L. (2012) Staging of the Cognitive Decline in Alzheimer's Disease: Insights from a Detailed Neuropsychological Investigation of Mild Cognitive Impairment and Mild Alzheimer's Disease. International Journal of Geriatric Psychiatry, 27, 423-432. 
[12] Perry, R.J. and Hodges, J.R. (1999) Attention and Executive Deficits in Alzheimer's Disease: A Critical Review. Brain, 122, 383-404. http://dx.doi.org/10.1093/brain/122.3.383

[13] Farias, S.T., Mungas, D., Reed, B.R., Harvey, D., Cahn-Weiner, D. and DeCarli, C. (2006) MCI Is Associated with Deficits in Everyday Functioning. Alzheimer Disease \& Associated Disorders, 20, 217-223. http://dx.doi.org/10.1097/01.wad.0000213849.51495.d9

[14] Perneczky, R., Pohl, C., Sorg, C., Hartmann, J., Tosic, N., Grimmer, T., Heitele, S. and Kurz, A. (2006) Impairment of Activities of Daily Living Requiring Memory or Complex Reasoning as Part of the MCI Syndrome. International Journal of Geriatric Psychiatry, 21, 158-162. http://dx.doi.org/10.1002/gps.1444

[15] Reppermund, S., Brodaty, H., Crawford, J.D., Kochan, N.A., Draper, B., Slavin, M.J., et al. (2013) Impairment in Instrumental Activities of Daily Living with High Cognitive Demand Is an Early Marker of Mild Cognitive Impairment: The Sydney Memory and Ageing Study. Psychological Medicine, 43, 2437-2445.

[16] Goldberg, T.E., Koppel, J., Keehlisen, L., Christen, E., Dreses-Werringloer, U., Conejero-Goldberg, C., Gordon, M.L. and Davies, P. (2010) Performance-Based Measures of Everyday Function in Mild Cognitive Impairment. American Journal of Psychiatry, 167, 845-853. http://dx.doi.org/10.1176/appi.ajp.2010.09050692

[17] Bangen, K.J., Jak, A.J., Schiehser, D.M., Delano-Wood, L., Tuminello, E., Han, S.D., et al. (2010) Complex Activities of Daily Living Vary by Mild Cognitive Impairment Subtype. Journal of the International Neuropsychological Society, 16, 630-639. http://dx.doi.org/10.1017/S1355617710000330

[18] Allaire, J.C., Gamaldo, A., Ayotte, B.J., Sims, R. and Whitfield, K. (2009) Mild Cognitive Impairment and Objective Instrumental Everyday Functioning: The Everyday Cognition Battery Memory Test. Journal of the American Geriatrics Society, 57, 120-125. http://dx.doi.org/10.1111/j.1532-5415.2008.02054.x

[19] Mioshi, E., Kipps, C.M., Dawson, K., Mitchell, J., Graham, A. and Hodges, J.R. (2007) Activities of Daily Living in Frontotemporal Dementia and Alzheimer Disease. Neurology, 68, 2077-2084. http://dx.doi.org/10.1212/01.wnl.0000264897.13722.53

[20] Dangour, A.D., Allen, E., Richards, M., Whitehouse, P. and Uauy, R. (2010) Design Considerations in Long-Term Intervention Studies for the Prevention of Cognitive Decline or Dementia. Nutrition Reviews, 68, S16-S21. http://dx.doi.org/10.1111/j.1753-4887.2010.00330.x

[21] de Jager, C.A., Hogervorst, E., Combrinck, M. and Budge, M.M. (2003) Sensitivity and Specificity of Neuropsychological Tests for Mild Cognitive Impairment, Vascular Cognitive Impairment and Alzheimer's Disease. Psychological Medicine, 33, 1039-1050. http://dx.doi.org/10.1017/S0033291703008031

[22] de Jager, C.A. and Budge, M.M. (2005) Stability and Predictability of the Classification of Mild Cognitive Impairment as Assessed by Episodic Memory Test Performance over Time. Neurocase, 11, 72-79. http://dx.doi.org/10.1080/13554790490896820

[23] Hogervorst, E., Combrinck, M., Lapuerta, P., Rue, J., Swales, K. and Budge, M. (2002) The Hopkins Verbal Learning Test and Screening for Dementia. Dementia and Geriatric Cognitive Disorders, 13, 13-20. http://dx.doi.org/10.1159/000048628

[24] Kramer, J.H., Jurik, J., Sha, S.J., Rankin, K.P., Rosen, H.J., Johnson, J.K. and Miller, B.L. (2003) Distinctive Neuropsychological Patterns in Frontotemporal Dementia, Semantic Dementia, and Alzheimer Disease. Cognitive \& Behavioral Neurology, 16, 211-218. http://dx.doi.org/10.1097/00146965-200312000-00002

[25] Libon, D.J., Massimo, L., Moore, P., Coslett, H.B., Chatterjee, A., Aguirre, G.K., et al. (2007) Screening for Frontotemporal Dementias and Alzheimer's Disease with the Philadelphia Brief Assessment of Cognition: A Preliminary Analysis. Dementia and Geriatric Cognitive Disorders, 24, 441-447. http://dx.doi.org/10.1159/000110577

[26] Pendlebury, S.T., Markwick, A., de Jager, C.A., Zamboni, G., Wilcock, G.K. and Rothwell, P.M. (2012) Differences in Cognitive Profile between TIA, Stroke and Elderly Memory Research Subjects: A Comparison of the MMSE and MoCA. Cerebrovascular Diseases, 34, 48-54. http://dx.doi.org/10.1159/000338905

[27] Perry, R.J. and Hodges, J.R. (2000) Differentiating Frontal and Temporal Variant Frontotemporal Dementia from Alzheimer's Disease. Neurology, 54, 2277-2284. http://dx.doi.org/10.1212/WNL.54.12.2277

[28] de Jager, C.A., Honey, T.E., Birks, J. and Wilcock, G.K. (2010) Retrospective Evaluation of Revised Criteria for the Diagnosis of Alzheimer's Disease Using a Cohort with Post-Mortem Diagnosis. International Journal of Geriatric Psychiatry, 25, 988-997. http://dx.doi.org/10.1002/gps.2448

[29] Huppert, F.A., Brayne, C., Gill, C., Paykel, E.S. and Beardsall, L. (1995) CAMCOG-A Concise Neuropsychological Test to Assist Dementia Diagnosis: Socio-Demographic Determinants in an Elderly Population Sample. British Journal of Clinical Psychology, 34, 529-541. http://dx.doi.org/10.1111/j.2044-8260.1995.tb01487.x

[30] Roth, M., Tym, E., Mountjoy, C.Q., Huppert, F.A., Hendrie, H., Verma, S. and Goddard, R. (1986) CAMDEX. A Standardised Instrument for the Diagnosis of Mental Disorder in the Elderly with Special Reference to the Early Detection of Dementia. British Journal of Psychiatry, 149, 698-709. http://dx.doi.org/10.1192/bjp.149.6.698 
[31] Folstein, M.F., Folstein, S.E. and McHugh, P.R. (1975) "Mini-Mental State". A Practical Method for Grading the Cognitive State of Patients for the Clinician. Journal of Psychiatric Research, 12, 189-198. http://dx.doi.org/10.1016/0022-3956(75)90026-6

[32] Brandt, J., Spencer, M. and Folstein, M. (1988) The Telephone Interview for Cognitive Status. Neuropsychiatry, Neuropsychology, \& Behavioral Neurology, 1, 111-117.

[33] Morris, J.C. (1997) Clinical Dementia Rating: A Reliable and Valid Diagnostic and Staging Measure for Dementia of the Alzheimer Type. International Psychogeriatrics, 9, 173-176. http://dx.doi.org/10.1017/S1041610297004870

[34] Yesavage, J.A., Brink, T.L., Rose, T.L., Lum, O., Huang, V., Adey, M. and Otto Leirer, V. (1982) Development and Validation of a Geriatric Depression Screening Scale: A Preliminary Report. Journal of Psychiatric Research, 17, 37-49. http://dx.doi.org/10.1016/0022-3956(82)90033-4

[35] Ferris, S.H., Lucca, U., Mohs, R., Dubois, B., Wesnes, K., Erzigkeit, H., et al. (1997) Objective Psychometric Tests in Clinical Trials of Dementia Drugs. Position Paper from the International Working Group on Harmonization of Dementia Drug Guidelines. Alzheimer Disease and Associated Disorders, 11, 34-38.

[36] Rosen, W.G., Mohs, R.C. and Davis, K.L. (1984) A New Rating Scale for Alzheimer's Disease. American Journal of Psychiatry, 141, 1356-1364.

[37] Cambridge Cognition Ltd. (2013) Cambridge Cognition-The Home of CANTAB. CANTAB.com [Internet]. Cambridge Cognition Ltd., Cambridge, c2013. http://www.cantab.com/

[38] Galasko, D., Bennett, D., Sano, M., Ernesto, C., Thomas, R., Grundman, M. and Ferris, S. (1997) An Inventory to Assess Activities of Daily Living for Clinical Trials in Alzheimer's Disease. Alzheimer Disease \& Associated Disorders, 11, S33-S39. http://dx.doi.org/10.1097/00002093-199700112-00005

[39] Sunderland, A., Harris, J. and Baddeley, A. (1983) Do Laboratory Tests Predict Everyday Memory? A Neuropsychological Study. Journal of Verbal Learning and Verbal Behavior, 22, 341-357. http://dx.doi.org/10.1016/S0022-5371(83)90229-3

[40] Graham, D., Cully, J.A., Snow, A.L., Massman, P. and Doody, R. (2004) The Alzheimer's Disease Assessment ScaleCognitive Subscale: Normative Data for Older Adults Controls. Alzheimer Disease and Associated Disorders, 18, $236-$ 240.

[41] Grundman, M., Petersen, R.C., Ferris, S.H., Thomas, R.G., Aisen, P.S., Bennett, D.A., et al. (2004) Mild Cognitive Impairment Can Be Distinguished from Alzheimer Disease and Normal Aging for Clinical Trials. JAMA Neurology, 61, 59-66. http://dx.doi.org/10.1001/archneur.61.1.59

[42] Royall, D.R., Cordes, J.A. and Polk, M. (1998) CLOX: An Executive Clock Drawing Task. Journal of Neurology, Neurosurgery \& Psychiatry, 64, 588-594. http://dx.doi.org/10.1136/jnnp.64.5.588

[43] Smith, A. (1968) The Symbol-Digit Modalities Test: A Neuropsychologic Test of Learning and Other Cerebral Disorders. In: Learning Disorders, Special Child Publications, Seattle, 83-91.

[44] Robertson, I.H., Thames Valley Test Company (1994) The Test of Everyday Attention. Thames Valley Test Company.

[45] Brandt, J., Aretouli, E., Neijstrom, M.S., Samek, J., Manning, K., Albert, M.S., et al. (2009) Selectivity of Executive Function Deficits in Mild Cognitive Impairment. Neuropsychology, 23, 607-618. http://dx.doi.org/10.1037/a0015851

[46] Papp, K.V., Snyder, P.J., Maruff, P., Bartkowiak, J. and Pietrzak, R.H. (2011) Detecting Subtle Changes in Visuospatial Executive Function and Learning in the Amnestic Variant of Mild Cognitive Impairment. PLoS ONE, 6, e21688. http://dx.doi.org/10.1371/journal.pone.0021688

[47] Voss, S. and Bullock, R. (2004) Executive Function: The Core Feature of Dementia? Dementia and Geriatric Cognitive Disorders, 18, 207-216. http://dx.doi.org/10.1159/000079202

[48] Brandt, J. (1991) The Hopkins Verbal Learning Test: Development of a New Memory Test with Six Equivalent Forms. Clinical Neuropsychologist, 5, 125-142. http://dx.doi.org/10.1080/13854049108403297

[49] Cano, S.J., Posner, H.B., Moline, M.L., Hurt, S.W., Swartz, J., Hsu, T. and Hobart, J.C. (2010) The ADAS-Cog in Alzheimer's Disease Clinical Trials: Psychometric Evaluation of the Sum and Its Parts. Journal of Neurology, Neurosurgery \& Psychiatry, 81, 1363-1368. http://dx.doi.org/10.1136/jnnp.2009.204008

[50] Petersen, R.C., Smith, G.E., Waring, S.C., Ivnik, R.J., Tangalos, E.G. and Kokmen, E. (1999) Mild Cognitive Impairment: Clinical Characterization and Outcome. JAMA Neurology, 56, 303-308. http://dx.doi.org/10.1001/archneur.56.3.303

[51] Winblad, B., Palmer, K., Kivipelto, M., Jelic, V., Fratiglioni, L., Wahlund, L.O., et al. (2004) Mild Cognitive Impairment-Beyond Controversies, towards a Consensus: Report of the International Working Group on Mild Cognitive Impairment. Journal of Internal Medicine, 256, 240-246. http://dx.doi.org/10.1111/j.1365-2796.2004.01380.x

[52] Marshall, D.A., Rentz, D.M., Frey, M.T., Locascio, J.J., Johnson, K.A. and Sperling, R.A., the Alzheimer's Disease Neuroimaging Initiative (2011) Executive Function and Instrumental Activities of Daily Living in Mild Cognitive Im- 
pairment and Alzheimer's Disease. Alzheimer's \& Dementia, 7, 300-308. http://dx.doi.org/10.1016/j.jalz.2010.04.005

[53] Perry, R.J. and Hodges, J.R. (2000) Relationship between Functional and Neuropsychological Performance in Early Alzheimer Disease. Alzheimer Disease \& Associated Disorders, 14, 1-10. http://dx.doi.org/10.1097/00002093-200001000-00001

[54] Cornish, I.M. (2000) Factor Structure of the Everyday Memory Questionnaire. British Journal of Psychology, 91, 427438. http://dx.doi.org/10.1348/000712600161916

[55] Salthouse, T.A. and Babcock, R.L. (1991) Decomposing Adult Age Differences in Working Memory. Developmental Psychology, 27, 763-776. http://dx.doi.org/10.1037/0012-1649.27.5.763

[56] Anderson, E., De Jager, C. and Iversen, S. (2006) The Placing Test: Preliminary Investigations of a Quick and Simple Memory Test Designed to Be Sensitive to Pre-Dementia Alzheimer's Disease but Not to Normal Ageing. Journal of Clinical and Experimental Neuropsychology, 28, 843-858. http://dx.doi.org/10.1080/13803390591001016

[57] McKenna, P. and Warrington, E.K. (1980) Testing for Nominal Dysphasia. Journal of Neurology, Neurosurgery \& Psychiatry, 43, 781-788. http://dx.doi.org/10.1136/jnnp.43.9.781 


\section{Online Supplement}

\section{Measures}

\subsection{Screening and Diagnostic Assessments}

\subsubsection{Telephone Interview for Cognitive Status-Modified (TICS-M) [32]}

The TICS-M is a cognitive function test, developed as a dementia screen, and is suitable for administration by telephone. The 13-item version of the test was used, sensitive to early cognitive impairment, in which a higher proportion of the total score (maximum score 39 points) is contributed to by tasks of immediate and delayed recall, than in other screening tools, such as the MMSE or CAMCOG. It also includes tasks of orientation, attention, semantic memory, and comprehension and language ability. The total score and the Word List Recall score (WLR; total words recalled on immediate and delayed memory tasks) were used as indicators of cognitive status for screening and diagnostic purposes. Higher Total and WLR scores indicate better performance.

\subsubsection{Mini Mental State Examination (MMSE) [31]}

The MMSE is a cognitive screening instrument which assesses cognitive functioning in five areas: Orientation, Registration, Recall, Attention/Concentration/Calculation, and Language, with each subtest area contributing approximately equal weight to the total score. The maximum total score is 30 points, where higher scores indicate less impairment.

\subsubsection{Subjective Memory Complaint (SMC) Questions (Selected Questions from the Cambridge Examination for Mental Disorders of the Elderly (CAMDEX)) [30]}

A measure of participants' subjective accounts of memory impairment was assessed with questions assessing memory complaints from the CAMDEX. Four questions were administered, including those assessing participants' subjective ability to remember names of people they know well and whether they had been lost in familiar places. The SMC was scored dichotomously, that is, SMC present or absent, where present indicated endorsement of 2 or more items as SMC items. Note that this measure was not used in the diagnostic classification of $\mathrm{AD}$ participants, as reduced insight may mask subjective reporting of memory difficulties.

\subsubsection{Geriatric Depression Scale (GDS) [34]}

The GDS is a 30-item depression screen for older adults, covering symptoms such as interest and enjoyment in activities, energy levels and hopefulness regarding the future, where scores of 0 - 9 indicate normal mood, 10 19 mild depression, and 20 - 30 severe depression.

\subsubsection{Clinical Dementia Rating (CDR) [33]}

The CDR provides a measure of clinical severity of dementia, covering six areas of cognitive and functional ability: Memory, Orientation, Judgment and Problem-solving, Community Affairs, Home and Hobbies, and Personal Care. Performance in these areas is rated on a 5-point scale (Personal care, on a 4-point scale) on the basis of information gathered by means of clinical interview with the participant and a reliable informant. These scores then contribute, using a mathematical algorithm, to the calculation of an overall CDR score, which provides a measure of dementia severity on a 5-point scale: 0 indicates no impairment, $0.5,1,2$, and 3 indicate dementia of increasing severity, that is, very mild, mild, moderate, and severe, respectively. A CDR-SoB score is also calculated from the sum of all the individual scores for each domain. CDR ratings were made by consensus decision of at least two research neuropsychologists or nurses.

\subsection{Functional Assessments}

\subsubsection{Alzheimer's Disease Cooperative Study-Activities of Daily Living (ADCS-ADL) [38]}

This measure assesses elderly participants' ability to carry out routine activities in everyday life pertaining to personal care, household maintenance, communicating and interaction, hobbies and interests, and decisionmaking and judgement ability. Administered to an informant or caregiver, functioning in the above areas is rated on 23 items as performed in the previous 4-week period, with each item rated according to the level of independence with which the task can be performed (from "can perform task independently" to "needs physical help"). Total scores range from 0 to 78 , with higher scores indicating less impairment. The items are grouped 
into basic ADL (personal care) and instrumental ADL (household maintenance, communicating and interaction, hobbies and interests, and decision-making and judgement ability). Used with permission from the NIA Alzheimer's Disease Cooperative Study (NIA Grant AG10483).

\subsubsection{Everyday Memory Questionnaire (EMQ) [39]}

The EMQ is a 35-item questionnaire which assesses memory impairment in 5 domains: speech, reading and writing, faces and places, actions, and learning new things. The items have been shown to load onto 5 factors, namely retrieval, task monitoring, conversational monitoring, spatial memory and memory for activities [54]. Each item is ranked on a 5-point scale ( 0 - 4 points) which indicates the frequency with which a particular memory difficulty occurs, ranging from "Never" to "Several times in a day". EMQ score ranges from 0 to 140 , where a higher score indicates greater impairment. A self-report version of the questionnaire was completed by HE participants, and an informant version was completed when participants were in the aMCI or AD groups to avoid issues of reduced insight into memory difficulties. If completed by the informant, the EMQ was filled in following completion of the ADCS-ADL.

\subsection{Cognitive Assessment Battery}

\subsubsection{Alzheimer's Disease Assessment Scale-Cognitive Subscale (ADAS-Cog) [36]}

The ADAS-Cog is widely used in clinical trials as a measure of cognitive impairment or change. The total score (maximum of 70) provides an overall measure of cognitive functioning, with higher scores indicating greater impairment. This score is derived from performance on the subtests which comprise the scale, including tasks of memory, praxis, orientation, and language. An additional task of executive functioning, the Maze task, derived from the Alzheimer's Disease Cooperative Study (ADCS) extended version of the ADAS was included as part of the ADAS-Cog in this study. The Maze task is scored on the number of seconds it takes the participant to complete the task; therefore higher scores indicate greater impairment. Three versions of the ADAS-Cog were administered; one at each of the study visits. Across the three versions, the stimuli for the word recall/recognition and the Maze task were varied. The more comprehensively assessed CAMCOG Orientation sub-test was used in this study in place of the ADAS-Cog Orientation sub-test. The ADAS-Cog was administered to aMCI and AD subjects only. Normative data from the literature.was used for the HE for the purpose of calculating $\mathrm{z}-$ scores [40] [41].

\subsubsection{Cambridge Automated Neuropsychological Test Battery (CANTAB) [37]}

Three tasks from the computerized CANTAB battery were administered: Paired Associate Learning (PAL), Reaction Time Index (RTI), and Spatial Working Memory (SWM). Responses on each of these tasks were made by the participant on a touch-screen computer.

1) PAL

The PAL is a test of episodic memory in the visuospatial modality. In this task, six boxes appear on the screen, followed by the presentation of a series of visual patterns. Each pattern is shown at a different location (inside one of the six boxes), and following presentation of the series, participants must respond as to which pattern was presented in which location. Patterns were included on the basis of being difficult to encode verbally. There are six levels of difficulty, or stages - constituted by an increased number of patterns that must be encoded and recalled $(2,3,4,5,6,8$ pattern). The test is discontinued after failure to correctly identify the location of all patterns on up to 6 consecutive trials at each stage. Two main scores were calculated: total number of errors and number of levels/stages reached. An adjusted total score allowing for predicted errors on levels not completed is calculated for those who discontinue the task early.

2) RTI

The RTI test provides a measure of attention and processing speed by means of two tasks of a) simple and b) 5-choice reaction time. In this test, participants must hold down a button, and in two separate conditions, respond either to the appearance of a yellow spot stimulus within a single circle or location by touching the screen at this location (simple reaction time task), or to this same spot when it appears at one of five possible locations (5-choice reaction time). Several measures are recorded: simple and 5-choice reaction time, and simple and 5choice movement time. Simple reaction time is the speed in milliseconds it takes the participant to release the press pad in response to the stimulus appearing in a single location. Five-choice reaction time is the speed in 
milliseconds it takes the subject to release the press pad in response to the stimulus appearing in any one of five locations. Simple movement time is the time taken to touch the stimulus after the press pad is released and in trials where stimuli appear in one location only. Five-choice movement time is the time taken to touch the stimulus after the press pad has been released and in trials where one of five possible different stimuli have been presented.

3) SWM

SWM measures one's ability to retain and manipulate spatial information in working memory. In this task, an array of colored boxes appears on the screen, and the participant must search for a number of blue tokens hidden inside these boxes. Only one token is hidden at a time, and participants must find enough tokens within a particular trial, to fill an empty space on the right on the screen. The number of tokens to be found increases with subsequent trials and importantly, within each trial, once a token has been found at a particular location the participants must not search that box for a further token as this constitutes an error. Task instructions are given, but following this, participants are left to complete the task without guidance, meaning assessment of their strategy or approach to completing the task can also be assessed. Two main scores are calculated: SWM Errors and SWM Errors at 6 box stage.

\subsection{Additional OPTIMA Battery Tasks}

\subsubsection{Cambridge Examination for Mental Disorders of the Elderly-Cognitive Examination (CAMCOG) [30]}

The CAMCOG is a pencil-and-paper test assessing cognitive function in the domains of orientation, language, memory and learning, attention and calculation, abstract thinking, praxis, and perception. Some task items included in the CAMCOG overlap directly with other cognitive assessments included in the study battery (e.g. the MMSE), thus, where this occurred, items were not readministered as part of the CAMCOG. Alternate versions of verbal recall and recognition (e.g. recalling address details), coin recognition and calculation items were administered to avoid repetition and learning effects from the previous testing visit. The CAMCOG provides a measure of overall cognitive functioning by means of a total score, maximum 107 points (where a higher score indicates better performance), and measures of various cognitive domains by means of subscale scores. Of particular interest for this study was the CAMCOG Learning score, which provides a measure of learning memory (maximum score 17 points), where higher scores indicate more intact learning ability.

\subsubsection{Hopkins Verbal Learning Test Revised (HVLT) [48]}

The HVLT is a test of verbal immediate and delayed recall and recognition memory. Participants are read a list of 12 words on three occasions (trials 1 - 3) and must recall as many words as possible after each presentation. The HVLT Total Recall (HVLT TR) score (maximum 36) is the total number of words recalled across the three trials. The Learning Index score gives an indication of the amount of learning that occurs over subsequent presentations of the word list, and is calculated by subtracting the number of words correctly recalled on trial 1 from the higher of the total of words correctly recalled on trial 2 or 3 . After a delay of approximately 20 minutes, participants are again asked to recall as many words as possible from the 12-word list. The HVLT delayed recall (HVLT DR) score is the number of words (maximum 12) correctly recalled on this trial. A recognition trial is then administered where 24 words are read, and the participant must identify those which appeared in the original 12-word list. The Discrimination Index is the number of true positive words recognised minus false positive endorsements from the recognition condition. An alternate version of this task was administered on each study visit.

\subsubsection{Pattern and Letter Comparison Test [55]}

The Pattern and Letter Comparison task is a test of processing speed. In the Pattern comparison subtest, participants are shown pairs of line drawings and have to respond as to whether the patterns in each pair are the same or different, responding correctly to as many pairs as possible within a time limit of 20 seconds. In the Letter comparison subtest, the premise is the same, but in this case, participants are responding as to whether strings of letters, arranged in pairs, are the same or different as quickly as possible, also within a time limit of 20 seconds. The total score for each subtest is the total number of correct responses made within the time limit (maximum of 30 points for Pattern comparison, maximum of 21 points for Letter comparison per page). 


\subsubsection{The Placing Test (TPT) [56]}

The Placing Test is a test of visuospatial paired associates learning. Participants are shown 12 pictures of objects (e.g. a vase, a picnic basket). Items are arranged across 6 pages, each divided into a $2 \times 2$ grid. Two picture stimuli appear on each page, the locations of which vary randomly, while the remaining grid positions remain blank. Before starting the task, participants are told that the grid quadrants are numbered $1-4$ (top left $=1$, top right $=2$, bottom left $=3$, bottom right $=4$ ). To ensure full attention to the stimulus set, participants are asked to say whether the objects are "pleasant", "unpleasant", or "neutral", and to respond "blank" to empty quadrants. The administrator points to each of the quadrants in order, asking the participant to respond as outlined. When all 6 pages have been administered, participants are shown a further page, also in the above grid formation, with no picture stimuli, but with the squares numbered as above. Each of the individual picture stimuli is now shown to the participant, and they must respond as to which square $(1-4)$ they previously saw the stimulus appear in. The total score is the number of object locations correctly identified.

\subsubsection{Symbol Digit Modalities Test (SDMT) [43]}

The SDMT provides a measure of attention and information processing. Participants are shown a key comprised of a line of symbols, each of which has a number which corresponds to it. Participants are then presented with a series of symbols that do not have their corresponding number attached. They must use the key to fill in the missing numbers for as many symbols as possible within a time limit of 90 seconds. The total score is the number of digits correctly entered against the series of symbols within the time limit, out of a possible maximum of 110 .

\subsubsection{Map Search Task (from Test of Everyday Attention) [44]}

The Map Search task is a test of visual scanning and selective attention in which participants are required to circle as many target stimuli as possible (from a total of 80) from amongst an array of different distractors on a busy road map within a time limit of two minutes. Three alternative versions of this task exist, one of which was used at each study visit. Target stimuli include petrol stations, garages and restaurants, symbolized by pictures of a petrol pump, spanner and cutlery, including distractor stimuli such as shopping trolleys. The total score is the number of target stimuli circled within the time limit (maximum 80).

\subsubsection{Graded Naming Test (GNT) [57]}

The GNT is a test of semantic memory, where participants are asked to give the name of a series of 30 black and white line drawings of objects. Test items are of varying familiarity (and thus difficulty), with less familiar objects presented later in the test. The total score is the number of objects correctly named (maximum 30 points).

\subsubsection{CLOX [42]}

The CLOX task is a test of both constructional praxis and executive functioning. Participants are instructed to draw a clock that reads 1.45 , and to set the hands and numbers on the face so child could read them. They are then given a sheet of paper on which to do this, through which the outline of a circle printed on the opposite side of the page can be seen. Once this part of the test (CLOX 1) is completed, the test administrator then turns the page over, and in the printed circle, demonstrates drawing a clock, with correct placement, first of the 12, 3, 6, and 9, and then the remaining numbers and hands (drawn as arrows). The participant is then asked to draw a clock "just like" the administrators (CLOX 2), eliminating the executive element of the CLOX 1 condition; that is, the additional requirement of planning an approach to the task. Each part of the CLOX test is scored out of 15 points according to a set of criteria, and a dysexecutive score is calculated by subtracting the CLOX 2 from the CLOX 1 score.

\section{Acknowledgements Including Sources of Support}

The authors thank Audrey Rissmiller, Epidemiology, Merck \& Co., Inc., all the OPTIMA participants and study partners who agreed to take part in the study, and the OPTIMA nurses who helped with medical aspects of the study. The study was funded by Merck Sharp \& Dohme Corp., a subsidiary of Merck \& Co., Inc. Mark Marsico, Julie Chandler, April Grant and Xingshu Zhu are employees of Merck and own stock/stock options in Merck. Celeste de Jager and Arwen Markwick were paid for services conducted on behalf of Merck. 


\section{List of Abbreviations}

AD: Alzheimer's Disease

ADAS-Cog: Alzheimer's Disease Assessment Scale-Cognitive subscale

ADCS-ADL: Alzheimer's Disease Cooperative Study-Activities of Daily Living

ADL: Activities of Daily Living

aMCI: Amnestic Mild Cognitive Impairment

ANOVA: Analysis of Variance

CAMCOG: Cambridge Cognitive Examination

CAMDEX: Cambridge Examination for Mental Disorders of the Elderly

CANTAB: Cambridge Automated Neuropsychological Test Battery

CDR: Clinical Dementia Rating

CLOX 1: Clock Drawing Task 1

CPU: Central Processing Unit

EMQ: Everyday Memory Questionnaire

GDS: Geriatric Depression Scale

GNT: Graded Naming Test

HE: Health Elderly

HVLT TR: Hopkins Verbal Learning Test Total Recall

HVLT DR: Hopkins Verbal Learning Test Delayed Recall

LCS: Letter Comparison Speed

MCI: Mild Cognitive Impairment

MMSE: Mini-Mental State Examination

NINCDS-ADRDA: National Institute of Neurological and Communicative Disorders and Stroke-Alzheimer's

Disease and Related Disorders Association

OPTIMA: Oxford Project to Investigate Memory and Aging

PAL: Paired Associate Learning

PALTOTEA: Paired Associate Learning Total Errors Adjusted

PCS: Pattern Comparison Speed

RTI: Reaction Time Index

SD: Standard Deviation

SDMT: Symbol Digit Modalities Test

SMC: Subjective Memory Complaint

sRT: Simple Reaction Time

SWM: Spatial Working Memory

SWMBE: Spatial Working Memory between Errors

TICS-M: Telephone Interview for Cognitive Status-Modified

TPT: The Placing Test

5cRT: Five-Choice Reaction Time 
Scientific Research Publishing (SCIRP) is one of the largest Open Access journal publishers. It is currently publishing more than 200 open access, online, peer-reviewed journals covering a wide range of academic disciplines. SCIRP serves the worldwide academic communities and contributes to the progress and application of science with its publication.

Other selected journals from SCIRP are listed as below. Submit your manuscript to us via either submit@scirp.org or Online Submission Portal.
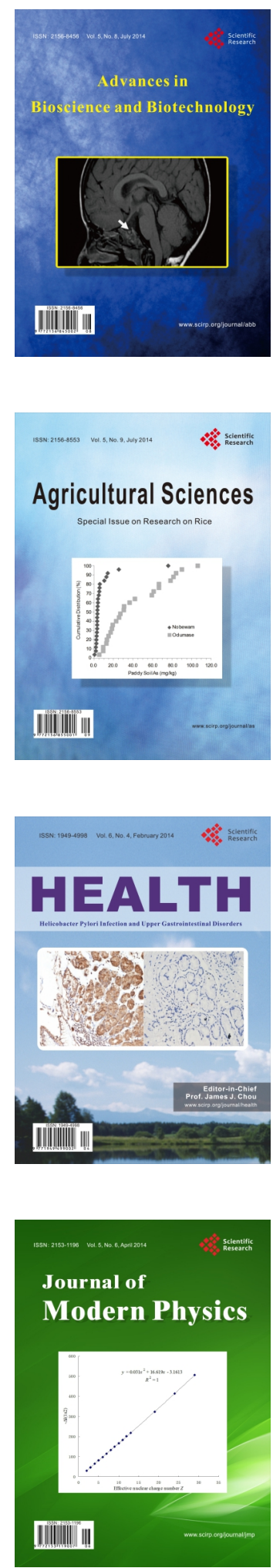
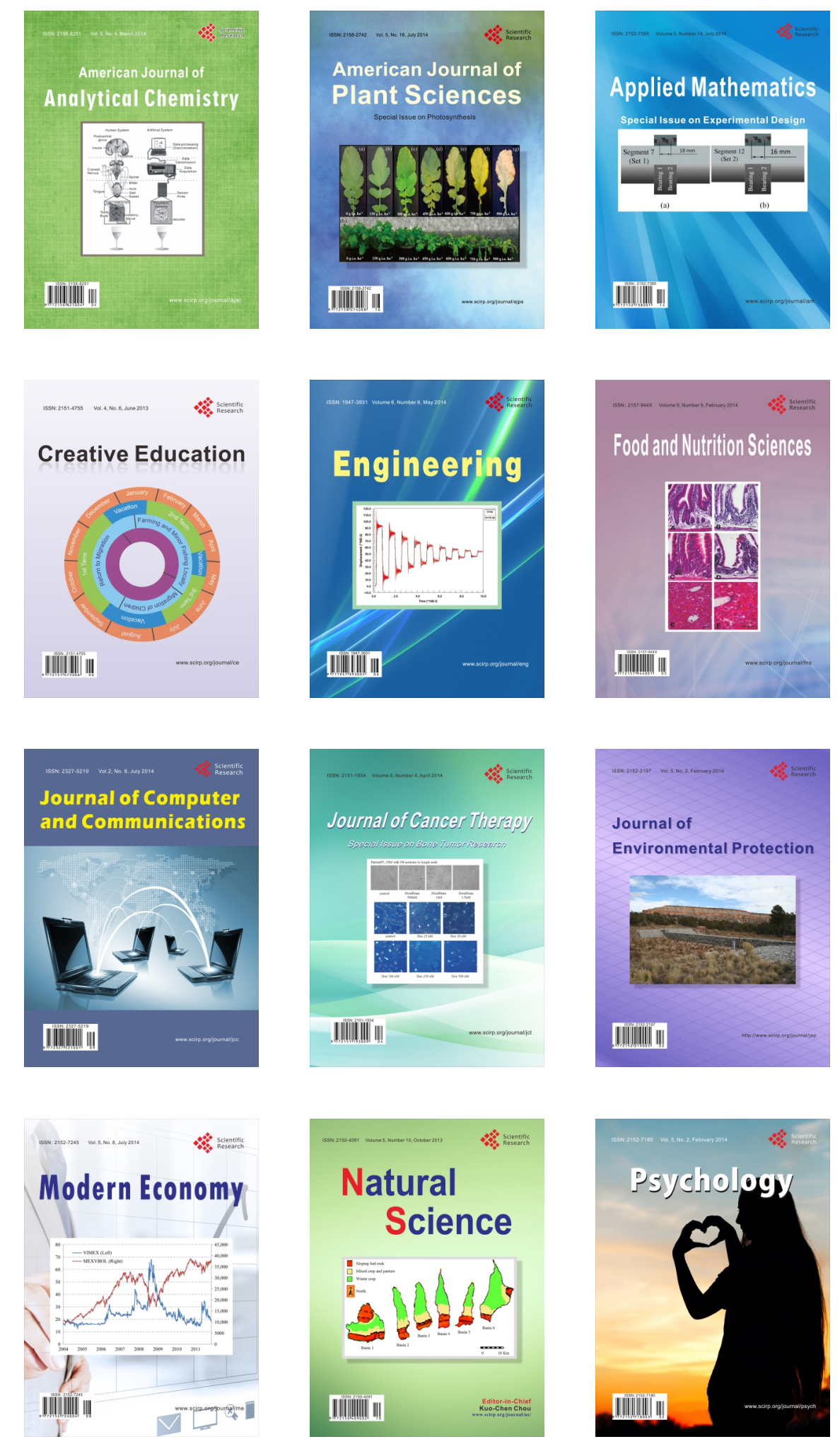